\section{Global Biodiversity Conservation Priorities}

\author{
T. M. Brooks, ${ }^{1,2,3 *}$ R. A. Mittermeier, ${ }^{1}$ G. A. B. da Fonseca, ${ }^{1,4}$ ]. Gerlach, ${ }^{5,6}$ M. Hoffmann, ${ }^{1}$ \\ J. F. Lamoreux, ${ }^{3}$ C. G. Mittermeier, ${ }^{1}$ ]. D. Pilgrim, $^{7}$ A. S. L. Rodrigues ${ }^{5}$
}

The location of and threats to biodiversity are distributed unevenly, so prioritization is essential to minimize biodiversity loss. To address this need, biodiversity conservation organizations have proposed nine templates of global priorities over the past decade. Here, we review the concepts, methods, results, impacts, and challenges of these prioritizations of conservation practice within the theoretical irreplaceability/vulnerability framework of systematic conservation planning. Most of the templates prioritize highly irreplaceable regions; some are reactive (prioritizing high vulnerability), and others are proactive (prioritizing low vulnerability). We hope this synthesis improves understanding of these prioritization approaches and that it results in more efficient allocation of geographically flexible conservation funding.

$\mathrm{H}$ uman actions are causing a biodiversity crisis, with species extinction rates up to 1000 times higher than background (1). Moreover, the processes driving extinction are eroding the environmental services on which humanity depends (2). People care most about what is close to them, so most responses to this crisis will be local or national (3). Thus, approximately $90 \%$ of the $\$ 6$ billion of annual conservation funding originates in and is spent within economically rich countries (4). However, this leaves globally flexible funding of hundreds of millions of dollars annually from multilateral agencies (such as the Global Environment Facility), bilateral aid, and private sources including environmentally focused corporations, foundations, and individuals. These resources are frequently the only ones available where conservation is most needed, given that biodiversity is unevenly distributed and the most biodiverse places are often the most threatened and poorest economically (5). Accordingly, geographically flexible resources exert disproportionate influence on conservation worldwide and have a key role in the recently agreed-upon intergovernmental 2010 target to reduce significantly the rate of biodiversity loss (6).

The development of strategies to best allocate globally flexible conservation resources has attracted considerable attention since the pioneering work of Myers (7), resulting in

${ }^{1}$ Conservation International, 1919 M Street, NW, Washington, DC 20036, USA. ${ }^{2}$ World Agroforestry Centre (ICRAF), Post Office Box 35024, University of the Philippines, Los Baños, Laguna 4031, Philippines. ${ }^{3}$ Department of Environmental Sciences, University of Virginia, Charlottesville, VA 22904, USA. ${ }^{4}$ Departamento de Zoologia, Universidade Federal de Minas Gerais, Belo Horizonte, MG 31270, Brazil. ${ }^{5}$ Department of Zoology, University of Cambridge, Downing Street, Cambridge CB2 3E], UK. ${ }^{6}$ Nature Protection Trust of Seychelles, Post Office Box 207, Victoria, Mahé, Seychelles. ${ }^{7}$ BirdLife International in Indochina, 4/209 Doi Can Street, Ba Dinh, Hanoi, Vietnam.

*To whom correspondence should be addressed. E-mail: t.brooks@conservation.org much progress as well as much controversy. The wide variety of approaches has led to criticism that there is duplication of effort and lack of clarity (8). Although attempts have been made to summarize conservation planning strategies by scale (9), none has done so within the framework of conservation planning (10). We review the published concepts and methods behind global biodiversity conservation prioritization, assess the remaining challenges, and highlight how this synthesis can inform allocation of globally flexible resources.

\section{Global Prioritization in Context} diversity conservation prioritization have been published over the past decade, each with involvement from nongovernmental organizations (fig. S1). Conceptually, they all fit within the framework of "irreplaceability" relative to "vulnerability" (Fig. 1), which is central to conservation planning theory (10). However, they map onto different portions of the framework: Most of the templates prioritize high irreplaceability, but some prioritize high vulnerability and others prioritize low vulnerability. These differences are key to understanding how and why the nine prioritizations differ, yielding priority maps that cover from less than one-tenth to more than a third of Earth's land surface (Fig. 2).

Six of the nine templates of global conservation priority incorporate irreplaceability-measures of spatial conservation options (10). The most common measure of irreplaceability is plant (11-14) or bird (15) endemism, often supported by terrestrial vertebrate endemism overall $(11,13,14)$. The logic for this is that greater the number of endemic species in a
Nine major institutional templates of global bio- region, the more biodiversity is lost if that region is lost (although, in a strict sense, any location with even one endemic species is irreplaceable). In addition to the number of endemic species, other aspects of irreplaceability have been proposed, including taxonomic uniqueness, unusual phenomena, and global rarity of major habitat types (16), but these remain difficult to quantify. Although species richness within a given area is popularly assumed to be important in prioritization, none of the approaches relies on species richness alone. This is because species richness is driven by common, widespread species; thus, strategies focused on species richness tend to miss exactly those biodiversity features most in need of conservation $(17,18)$. Three approaches do not incorporate irreplaceability (19-21).

The choice of irreplaceability measures is to some degree subjective, in that data limitations currently preclude the measurement of overall biodiversity. Furthermore, these data constraints mean that, with the exception of endemic bird areas (15), the measures of irreplaceability used in global conservation prioritization have been derived from the opinions of specialists. Subsequent tests of plant endemism estimates (22) have shown this expert opinion to be quite accurate. However, reliance on specialist opinion means that results cannot be replicated, raising questions concerning the transparency of the approaches $(8)$. It also prevents a formal measurement of irreplaceability, which requires the identities of individual biodiversity features, such as species names, rather than just estimates of their magnitude expressed as a number $(8,23)$.

Five of the templates of global conservation priority incorporate vulnerability - measures of temporal conservation options (10). A recent classification of vulnerability (24) recognizes four types of measures: (i) environmental and spatial variables, (ii) land tenure, (iii) threatened species, and (iv) expert opinion. Of these, environmental and spatial variables have been

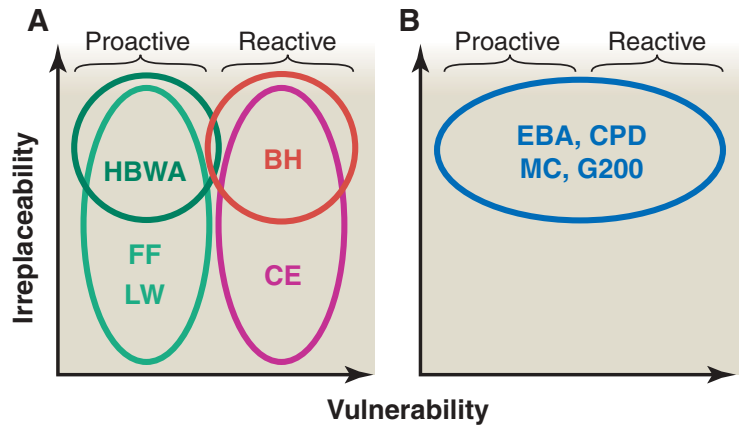

Fig. 1. Global biodiversity conservation priority templates placed within the conceptual framework of irreplaceability and vulnerability. Template names are spelled out in the Fig. 2 legend. (A) Purely reactive (prioritizing low vulnerability) and purely proactive (prioritizing high vulnerability) approaches. (B) Approaches that do not incorporate vulnerability as a criterion (all prioritize high irreplaceability). 
used most frequently in global conservation prioritization, measured as proportionate habitat loss $(11,14,20,21)$. Species-area relationships provide justification that habitat loss translates into biodiversity loss (1). However, the use of habitat loss as a measure of vulnerability has several problems: It is difficult to assess with the use of remote sensing for xeric and aquatic systems, it does not incorporate threats such as invasive species and hunting pressure, and it is retrospective rather than predictive (24). The frontier forests approach (19) uses absolute forest cover as a measure.

In addition to habitat loss, land tenuremeasured as protected area coverage - has also been incorporated into two approaches $(16,21)$. Other possible surrogates not classified by Wilson et al. (24) include human population growth and density, which are widely thought to be relevant $(25-27)$ and were integral to two of the systems $(14,20)$. None of the global conservation prioritization templates used threatened species or expert opinion as measures of vulnerability. Political and institutional capacity and governance (27) affect biodiversity indirectly, but have not been incorporated to date. This is true for climate change as well, which is of concern given that its impact is likely to be severe (28). Finally, although costs of conservation generally increase as the threat increases, no proposals for global biodiversity conservation priority have yet incorporated costs directly, despite the availability of techniques to do this at regional scales (29). Two of the tem- plates of global conservation prioritization do not incorporate vulnerability $(12,13)$, and the remaining two incorporate it only peripherally $(15,16)$.

The spatial units most commonly used in systematic conservation planning are equal-area grids. However, data limitations have precluded their use in the development of actual templates of global biodiversity conservation priority to date. Instead, all proposals, with the exception of megadiversity countries (13), are based on biogeographic units. Typically, these units are defined a priori by specialist perception of the distribution of biodiversity. For example, "ecoregions," one of the most commonly used such classifications, are "relatively large units of land containing a characteristic set of natural communities that share a large majority of their species, dynamics, and environmental conditions" (16). Only in the endemic bird areas approach are biogeographic units defined a posteriori by the distributions of the species concerned (15). Relative to equalarea grids, biogeographic units bring advantages of ecological relevance, whereas megadiversity countries (13) bring political relevance.

Reliance on biogeographic spatial units raises several complications. Various competing bioregional classifications are in use (30), and the choice of system has considerable repercussions for resulting conservation priorities. Furthermore, when unequally sized units are used, priority may be biased toward large areas as a consequence of species-area relationships. Therefore, assessment of global conservation priorities should factor out area, either by taking residuals about a best-fit line to a plot of species against area (18) or by rescaling numbers of endemic species with the use of a power function (23). Nevertheless, the use of a priori bioregional units for global conservation prioritization will be essential until data of sufficient resolution become available to enable the use of grids.

In Fig. 3, we map the overlay of the global biodiversity conservation priority systems into geographic space from the conceptual framework of Fig. 1. Figure 3A illustrates the large degree of overlap between templates that prioritize highly vulnerable regions of high irreplaceability: tropical islands and mountains (including montane Mesoamerica, the Andes, the Brazilian Atlantic forest, Madagascar, montane Africa, the Western Ghats of India, Malaysia, Indonesia, the Philippines, and Hawaii), Mediterranean-type systems (including California, central Chile, coastal South Africa, southwest Australia, and the Mediterranean itself), and a few temperate forests (the Caucasus, the central Asian mountains, the Himalaya, and southwest China). Highly vulnerable regions of lower irreplaceability (generally, the rest of the northern temperate regions) are prioritized by fewer approaches. Figure 3B shows a large amount of overlap between templates for regions of low vulnerability but high irreplaceability, in particular the three major tropical rainforests of Amazonia, the Congo, and New Guinea. Regions of simultaneously lower vulnerability and irreplaceability, such as
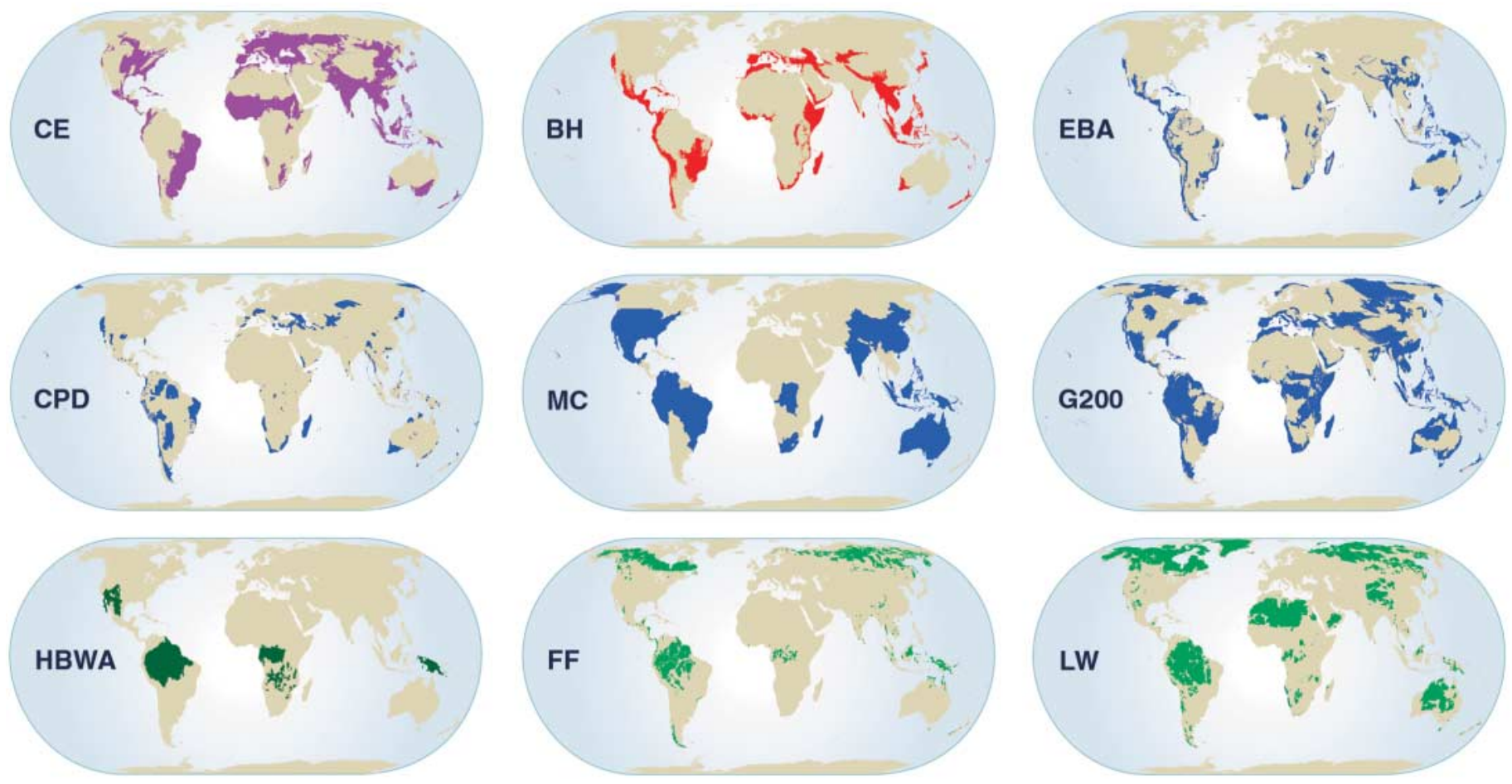

Fig. 2. Maps of the nine global biodiversity conservation priority templates: $\mathrm{CE}$, crisis ecoregions (21); $\mathrm{BH}$, biodiversity hot spots [(11), updated by (39)]; $E B A$, endemic bird areas (15); CPD, centers of plant diversity (12); MC, megadiversity countries (13); G200, global 200 ecoregions [(16), updated by (54)]; HBWA, high-biodiversity wilderness areas (14); FF, frontier forests (19); LW, last of the wild (20). 
the boreal forests of Canada and Russia, and the deserts of the western United States and central Asia, are prioritized less often.

Two general observations are apparent. First, most land (79\%) is highlighted by at least one of the prioritization systems. Second, despite this, a noticeable pattern emerges from the overlay of different approaches. There is significant overlap among templates that prioritize irreplaceable regions (11-16), among those that prioritize highly vulnerable regions $(11,21)$, and among those that prioritize regions of low vulnerability $(14,19,20)$, but not between approaches across each of these three general classes (table S1). This provides useful cross-verification of priority regions (31).

These patterns of overlap reflect two approaches to how vulnerability is incorporated into conservation in the broadest sense: reactive (prioritizing areas of high threat and high irreplaceability) and proactive (prioritizing areas of low threat but high irreplaceability). The former are considered the most urgent priorities in conservation planning theory (10) because unless immediate conservation action is taken within them, unique biodiversity will soon be lost. The latter are often de facto priorities, because the opportunities for conservation in these are considerable (32). Biodiversity conservation clearly needs both approaches, but the implementation of each may correspond to different methods. On the one hand, large-scale conservation initiatives may be possible in wilderness areas, such as the establishment of enormous protected areas (one example is the 3,800,000-ha Tumucumaque National Park, created in the Brazilian state of Amapá in 2003). On the other hand, finely tuned conservation will be essential in regions of simultaneously high irreplaceability

\section{A}
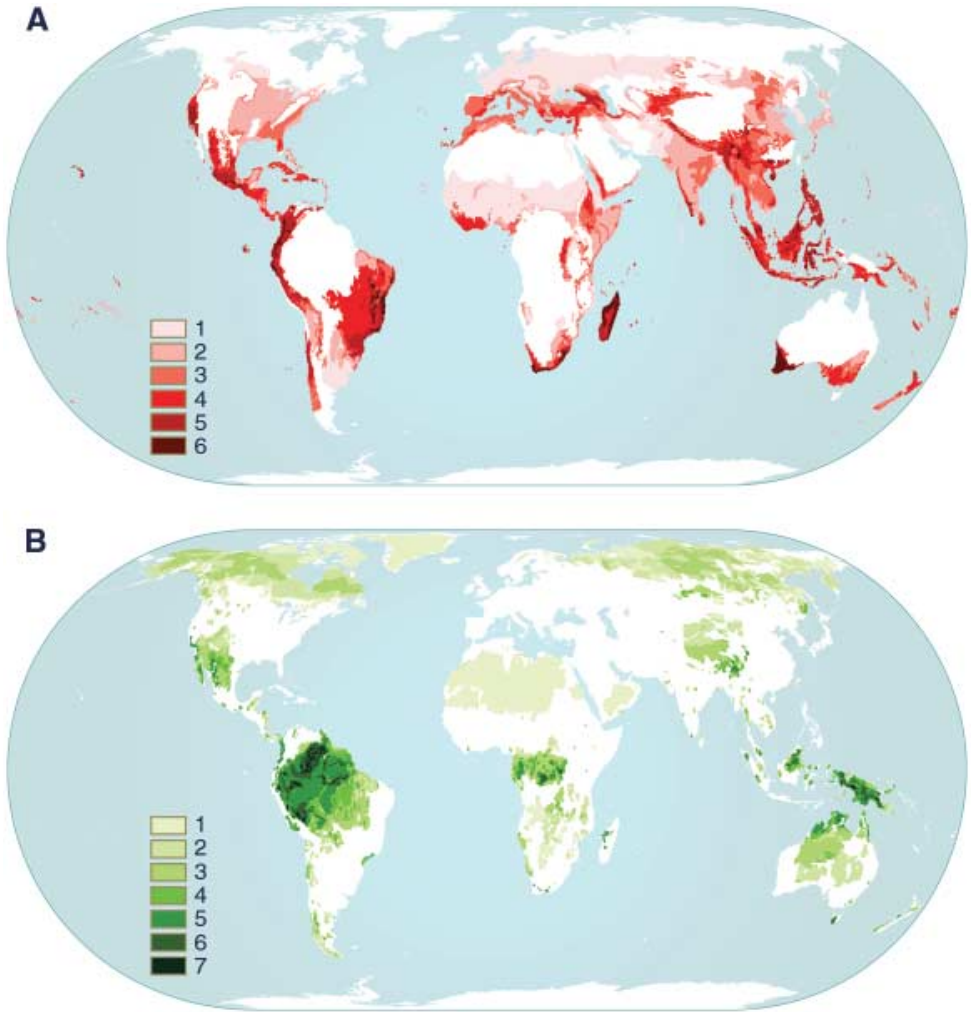

Fig. 3. Mapping the overlay of approaches prioritizing reactive and proactive conservation. (A) Reactive approaches, corresponding to the right-hand side of Fig. $1 \mathrm{~A}$, which prioritize regions of high threat, and those that do not incorporate vulnerability as a criterion (Fig. 1B); the latter are only mapped where they overlap with the former. (B) Proactive approaches, corresponding to the left-hand side of Fig. $1 \mathrm{~A}$, which prioritize regions of low threat, and those that do not incorporate vulnerability as a criterion (Fig. 1B); again, the latter are only mapped where they overlap with the former. Shading denotes the number of global biodiversity conservation prioritization templates that prioritize the shaded region, in both (A) and (B). and threat, where losing even

tiny patches of remnant habitat, such as the sites identified by the Alliance for Zero Extinction (33), would be tragic.

\section{Impact of Global Prioritization}

The appropriate measure of impact is the success of prioritization in achieving its main goal: influencing globally flexible donors to invest in regions where these funds can contribute most to conservation. Precise data are unavailable for all of the approaches (34), but hot spots alone have mobilized at least $\$ 750$ million of funding for
Both civil society and government organizations often use the recognition given to regions as global conservation priorities as justification when applying for geographically flexible funding. In addition, the global prioritization systems must have had sizeable effects in the cancellation, relocation, or mitigation of environmentally harmful activities, even in the absence of specific legislation. Unfortunately, resources still fall an order of magnitude short of required conservation funding (4). Nevertheless, the dollar amounts are impressive, and represent marked increases in conservation investment in these regions.

\section{Challenges Facing Global Prioritization} potential to reduce these constraints, particularly for , and amphibians (5). When these maps are combined with assessment of conservation status, they enable the development of threat metrics directly based on threatened species (36). So far, the main advances to global prioritization enabled by these new data are validation tests of existing templates (31). Encouragingly, global gap analysis of priorities for the representation of terrestrial vertebrate species in protected areas (36) and initial regional assessment of plants (37) yield results similar to existing approaches (fig. S2).

Invertebrates represent the bulk of eukaryotic diversity on Earth with more than a million known species and many more yet to be described (5). The conservation status of only $\sim 3500$ arthropods has been assessed (5), so global conservation priority is far from being able to incorporate megadiverse invertebrate taxa $(8,23)$. Although some regional data shows little overlap between priority areas for arthropods and those for plant and terrestrial vertebrate taxa (38), preliminary global data for groups such as tiger beetles and termites suggest much higher levels of congruence (39). Similarly, pioneering techniques to model overall irreplaceability by combining point data for megadiverse taxa with environmental data sets produce results commensurate with existing conservation priorities (40). These findings, although encouraging, in no way preclude the need to use primary invertebrate data in global conservation prioritization as they become available.

Aquatic systems feature poorly in existing conservation templates. Only one conservation prioritization explicitly incorporates aquatic systems (16). The most comprehensive study yet, albeit restricted to tropical coral reef ecosystems, identified 10 priority regions based on endemism and threat (41). Eight of these regions lie adjacent to priority regions highlighted in Fig. 3, raising the 
possibility of correspondence between marine and terrestrial priorities, despite the expectation that surrogacy of conservation priorities will be low between different environments (42). Efforts to identify freshwater priorities lag further behind, although initial studies reveal a highly uneven distribution of freshwater fish endemism (39).

Most measurement of irreplaceability is species based, raising the concern that phylogenetic diversity may slip through the net of global conservation priorities $(8,23,43)$. However, analyses for mammals (44) find that priority regions represent higher taxa and phylogenetic diversity better than would be predicted by the degree to which they represent species. Islands such as Madagascar and the Caribbean hold especially high concentrations of endemic genera and families (39). A heterodox perspective argues that the terminal tips of phylogenetic trees should be higher priorities than deep lineages (45). In any case, the balance of work implies that even if phylogenetic diversity is not explicitly targeted for conservation, global prioritization based on species provides a solid surrogate for evolutionary history.

That global conservation priority regions capture phylogenetic history does not necessarily mean that they represent evolutionary process (8). For example, transition zones or "biogeographic crossroads," frequently overlooked by conservation prioritization, could be of particular importance in driving speciation (46). On the other hand, there is evidence that areas of greatest importance in generating biodiversity are those of long-term climatic stability, especially where they occur in tropical mountains (47), which are incorporated in most approaches to global conservation prioritization. The development of metrics for the maintenance of evolutionary process is in its infancy and represents an emerging research front.

A final dimension that will prove important to assess in the context of global conservation prioritization concerns ecosystem services (43). Although the processes threatening biodiversity and ecosystem services are likely similar, the relationship between biodiversity per se and ecosystem services remains unresolved (48). Thus, while it is important to establish distinct goals for these conservation objectives (49), identification of synergies between them is strategically vital. This research avenue has barely been explored, and questions of how global biodiversity conservation priorities overlap with priority regions for carbon sequestration, climate stabilization, maintenance of water quality, minimization of outbreaks of pests and diseases, and fisheries, for example, remain unanswered. However, the correspondence between conservation priorities and human populations $(25,26)$ and poverty $(4,5)$ is an indication that the conservation of areas of high biodiversity priority will deliver high local ecosystem service benefits.

\section{From Global to Local Priorities}

The establishment of global conservation priorities has been extremely influential in directing resources toward broad regions. However, a number of authors have pointed out that global conservation prioritization has had little success in informing actual conservation implementation $(8,23)$. Separate processes are necessary to identify actual conservation targets and priorities at much finer scales, because even within a region as uniformly important as, for example, Madagascar, biodiversity and threats are not evenly distributed. Bottom-up processes of identification of priorities are therefore essential to ensure the implementation of area-based conservation (50).

Indeed, numerous efforts are underway to identify targets for conservation implementation. Many focus on the site scale, drawing on two decades of work across nearly 170 countries in the designation of important bird areas (51). There is an obvious need to expand such work to incorporate other taxa (52) and to prioritize the most threatened and irreplaceable sites (33). Such initiatives have recently gained strong political support under the Convention on Biological Diversity, through the development of the Global Strategy for Plant Conservation and the Programme of Work on Protected Areas. Both mechanisms call for the identification, recognition, and safeguarding of sites of biodiversity conservation importance. Meanwhile, considerable attention is also targeted at the scale of landscapes and seascapes to ensure not just the representation of biodiversity but also of the connectivity, spatial structure, and processes that allow its persistence (53).

Global conservation planning is key for strategic allocation of flexible resources. Despite divergence in methods between the different schemes, an overall picture is emerging in which a few regions, particularly in the tropics and in Mediterranean-type environments, are consistently emphasized as priorities for biodiversity conservation. It is crucial that the global donor community channel sufficient resources to these regions, at the very minimum. This focus will continue to improve if the rigor and breadth of biodiversity and threat data continue to be consolidated, which is especially important given the increased accountability demanded from global donors. However, it is through the conservation of actual sites that biodiversity will ultimately be preserved or lost, and thus drawing the lessons of global conservation prioritization down to a much finer scale is now the primary concern for conservation planning.

\section{References and Notes}

1. S. L. Pimm, G. J. Russell, J. L. Gittleman, T. M. Brooks, Science 269, 347 (1995).

2. Millennium Ecosystem Assessment, Ecosystems and Human Well-Being (Island Press, Washington, DC, 2005).

3. M. L. Hunter Jr., A. Hutchinson, Conserv. Biol. 8, 1163 (1994).

4. A. James, K. J. Gaston, A. Balmford, Nature 401, 323 (1999).

5. ]. E. M. Baillie et al., Global Species Assessment (IUCN, Gland, Switzerland, 2004)

6. A. Balmford et al., Science 307, 212 (2005).

7. N. Myers, Environmentalist 8, 187 (1988).
8. G. M. Mace et al., Nature 405, 393 (2000).

9. K. H. Redford et al., Conserv. Biol. 17, 116 (2003).

10. C. R. Margules, R. L. Pressey, Nature 405, 243 (2000)

11. N. Myers et al., Nature 403, 853 (2000).

12. WWF, IUCN, Centres of Plant Diversity (WWF and IUCN Gland, Switzerland, 1994-1997).

13. R. A. Mittermeier, P. Robles Gil, C. G. Mittermeier, Megadiversity (CEMEX, Mexico City, Mexico, 1997).

14. R. A. Mittermeier et al., Proc. Natl. Acad. Sci. U.S.A. 100 10309 (2003).

15. A. J. Stattersfield, M. J. Crosby, A. J. Long, D. C. Wege, Endemic Bird Areas of the World (BirdLife International, Cambridge, UK, 1998).

16. D. M. Olson, E. Dinerstein, Conserv. Biol. 12, 502 (1998).

17. C. D. L. Orme et al., Nature 436, 1016 (2005).

18. J. F. Lamoreux et al., Nature 440, 212 (2006).

19. D. Bryant, D. Nielsen, L. Tangley, Last Frontier Forests (World Resources Institute, Washington, DC, 1997).

20. E. W. Sanderson et al., Bioscience 52, 891 (2002).

21. J. M. Hoekstra et al., Ecol. Lett. 8, 23 (2005).

22. G. A. Krupnick, W. ]. Kress, Biodivers. Conserv. 12, 2237 (2003).

23. N. Brummitt, E. N. Lughadha, Conserv. Biol. 17, 1442 (2003)

24. K. Wilson et al., Environ. Manage. 35, 527 (2005).

25. R. P. Cincotta, J. Wisnewski, R. Engelman, Nature 404, 990 (2000)

26. A. Balmford et al., Science 291, 2616 (2001).

27. C. O'Connor, M. Marvier, P. Kareiva, Ecol. Lett. 6, 706 (2003).

28. C. Parmesan, G. Yohe, Nature 421, 37 (2003).

29. K. A. Wilson et al. Nature 440, 337 (2006).

30. P. Jepson, R. J. Whittaker, Conserv. Biol. 16, 42 (2002).

31. G. A. B. da Fonseca et al., Nature 405, 393 (2000).

32. M. Cardillo, G. M. Mace, ]. L. Gittleman, A. Purvis, Proc. Natl. Acad. Sci. U.S.A. 103, 4157 (2006).

33. T. H. Ricketts et al., Proc. Natl. Acad. Sci. U.S.A. $\mathbf{5 1}$ 18497 (2005).

34. B. S. Halpern et al., Conserv. Biol. 20, 56 (2006).

35. N. Myers, Bioscience 53, 916 (2003).

36. A. S. L. Rodrigues et al., Bioscience 54, 1092 (2004)

37. W. Küper et al., Ann. Missouri Bot. Gard. 91, 525 (2004).

38. A. P. Dobson, ]. P. Rodriguez, W. M. Roberts, D. S. Wilcove, Science 275, 550 (1997).

39. R. A. Mittermeier et al., Hotspots Revisited (CEMEX, Mexico City, Mexico, 2004).

40. S. Ferrier et al., Bioscience 54, 1101 (2004).

41. C. M. Roberts et al., Science 295, 1280 (2002).

42. W. V. Reid, Trends Ecol. Evol. 13, 275 (1998).

43. P. Kareiva, M. Marvier, Am. Sci. 91, 344 (2003).

44. W. Sechrest et al., Proc. Natl. Acad. Sci. U.S.A. 99, 2067 (2002).

45. T. L. Erwin, Science 253, 750 (1991).

46. T. B. Smith, R. K. Wayne, D. J. Girman, M. W. Bruford, Science 276, 1855 (1997).

47. ]. Fjeldså, ]. C. Lovett, Biodivers. Conserv. 6, 325 (1997).

48. M. Loreau et al., Science 294, 804 (2001).

49. S. Sarkar, Bioscience 49, 405 (1999).

50. R. J. Whittaker et al., Divers. Distrib. 11, 3 (2005).

51. BirdLife International, State of the World's Birds 2004 (BirdLife International, Cambridge, UK, 2004).

52. G. Eken et al., Bioscience 54, 1110 (2004).

53. R. M. Cowling, R. L. Pressey, M. Rouget, A. T. Lombard, Biol. Conserv. 112, 191 (2003).

54. D. M. Olson, E. Dinerstein, Ann. Missouri Bot. Gard. 89 199 (2002)

55. We thank G. Fabregas, D. Knox, T. Lacher, P. Langhammer N. Myers, and W. Turner for help with the manuscript, and the Gordon and Betty Moore Foundation for funding.

\section{Supporting Online Material}

www.sciencemag.org/cgi/content/full/313/5783/58/DC1

Figs. S1 and S2

Table $\mathrm{S1}$

References and Notes

10.1126/science. 1127609 